\title{
Improving the Life of the Wireless Sensor Network using Energy Harvesting Clustering
}

\author{
Nibedita Priyadarshini \\ Mohapatra \\ Asst. Professor \\ National Institute of Science \\ and Technology \\ Palur Hills, Berhampur, \\ Odisha
}

\author{
Jagruti Jena \\ Student (Btech Final year) \\ National Institute of Science \\ and Technology \\ Palur Hills, Berhampur, \\ Odisha
}

\author{
Sujata Kumari Sahu \\ Student (Btech Final year) \\ National Institute of Science \\ and Technology \\ Palur Hills, Berhampur, \\ Odisha
}

\begin{abstract}
In the modern era, Wireless sensor network (WSN) has seen new horizons among the research community. It has largest range of applications in most of the fields. Its beauty lies in its application area and it can be deployed anywhere. WSN has low cost, low power sensor nodes, can be deployed in large numbers and can be even used in hazardous environment. However, the sensor nodes in WSN do not have longer lifetime. Therefore, to meet this challenge of increasing the field lifetime of sensor nodes, the present study seeks to meet this end by using energy harvesting clustering model. Lifetime of the network increased largely by energy harvesting concept and clustering technique applied for effective use of energy. This increases the field lifetime of the sensor nodes before their complete degradation. In this way lifetime of the network improved a lot, which has seen through the experimental results in this paper.
\end{abstract}

\section{General Terms}

Wireless Sensor Networks, Energy harvesting.

\section{Keywords}

Cluster heads, energy consumption, power management.

\section{INTRODUCTION}

Wireless sensor networks (WSNs) lies on the top when we consider its popularity; it is possible due to its innovative and interesting applications in almost all fields ranging from environment monitoring to battlefield scenario. In computing and communication platforms, it sets a new level for monitoring different environments. It concerned to remote geographical area where human intervention is not possible [1, 2, and 3]. Every sensor node in WSN consists of four basic units namely sensor unit, transceiver unit, processor unit, power unit. The job of the sensor unit is to sense the particular environmental conditions such as temperature, pressure level, humidity level etc., again this sensed useful information converted to digital signal for communication purpose through an A-D converter.

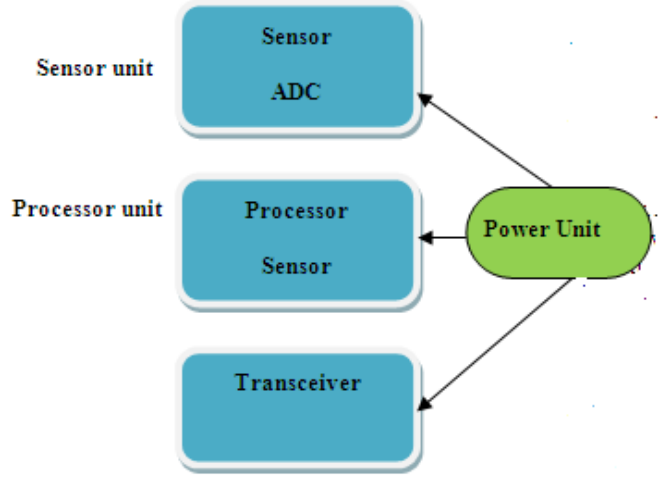

Fig 1.1Different units of a typical sensor node

The processor unit includes a microcontroller and memory unit. It saves the sensed data. The transceiver unit comprises of wireless radio transmitters and receiver stations. The power unit consists of batteries that provide necessary power to other units $[5,6]$. Many protocols proposed to reduce the energy consumption and thereby increase the lifetime of the sensor network. These protocols categorized into three types. The protocols coming under first class category focuses on controlling of transmission power at node level and at the same time maintain the connectivity of the network; it is possible by increasing the network capacity. However, here we have to compromise with the cost of the network. Protocols referred to next category make routing decisions based upon power optimization goals. Then protocols included in the last category make very important and useful decisions about topology control, that means which sensor nodes take part in the network activity (active mode) and which are not (sleep mode) taking part in the network operations at the same time. $[7,8,9,10]$. Here the nodes have the good knowledge about their respective positions through GPS or message passing.

\begin{tabular}{|cc|}
\hline $\begin{array}{c}\text { Available Energy } \\
\text { Consumable Energy (Eout) }\end{array}$ & (Eav) \\
\hline
\end{tabular}

Clustering techniques applied to reduce the energy consumption. These techniques also provide better load balancing, efficient resource utilization and data aggregation. As from analysis of different papers, many protocols propose in the direction of minimize energy consumption on relay paths to increase energy efficiency. Some proposes dynamic routing protocols in this context. Nevertheless, these approaches are not providing good results when some nodes are present in the most relaying path, it refers to popular path. As a result of this routing 
loop and unbounded delay are comes into picture. This clustering approach does not provide any assumptions about node capabilities and node distribution in the network. In the classic distributed analogy, a node can either sever or source, but both cannot be possible. When energy resource of a node depleted, that node is, a dead node or it fails to communicate. This is the motivation for rotating the server role among all the nodes present in the network. The research works with respect to WSN in the context of communication give a new direction to routing protocols. Which has given emphasis on to chose the routes for data transmission from the sensor node to base station in such a way that, the network lifetime maximized. It is not a permanent solution for maximize the lifetime of the network, it is helpful to some extent. As we know sensor nodes are battery powered, so designing of optimal routing protocol plays a very crucial role in WSN. Since battery, replacement is a crucial issue in most of the applications where sensor nodes deployed in more numbers. So the effort has to make the sensor network in efficient manner, the nodes get their energy from the environment itself where they deployed. In this way, it overcomes the battery limitations. It represented in another way we may call it as energy scavengers.

Energy harvesting can be possible through ambient power sources such as solar, thermal, mechanical radio frequency etc. Any of these sources shows time variant properties like pulse based behavior and frequency component variations. A very interesting fact of harvesting energy is that, the joint venture of harvesting source's random nature and communication process results the temporary depletion of energy storage unit of sensor nodes. Some research works find good solution, by using piezoelectric materials for conversion of strain energy from a structure into electrical energy to harvest energy to the sensor nodes in the network. This is a remarkable approach $[11,12,13]$.

It motivates a lot to work in this field. Many other researchers are also active in this area. There are different strategies to gain power from the respective environment. When rate of discharge is greater than the rate of charge then the particular system will run out of power. One interesting concept is that if the power consumption or power drawn by the sensing network minimized then the demand of power output from the harvester reduced to greater extent. To explain the perspective of energy utilization, the following energy utilization in WSN diagram presented here. The energy supplying system of WSN includes three parts, such as energy harvesting device, energy-consuming device and energy storage device. Arrow mark shows the energy flow and thickness degree of line indicates the rate of energy flow in the network.

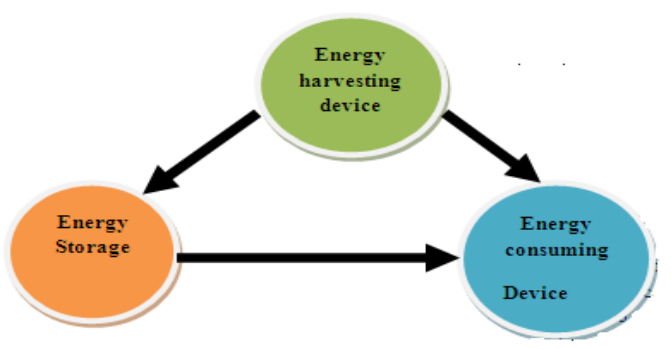

Fig 1.2Energy utilization in WSN
The function of the energy-harvesting device is to harvest environmental energy to power the sensor nodes in the network; it acts as producer. The role of the energyconsuming device is to utilize the harvested energy for supporting different workloads like sampling, communication and computation [14, 23] .The energy storage device perform the role of storing harvested energy from environment, it provides power to external sensor nodes. It has dual role, it can be either producer or consumer according to the current situation demands.

\section{PROBLEM STATEMENT}

To demonstrate the smart wireless sensing nodes capable of performing low power operation and to use radio frequency and efficient clustering technique to power these demonstrations. Here a novel model proposed, in which node has the capability to become a server (cluster head) or a sensor node. A particular node in the network only knows about the servers or cluster heads that are coming under its communication range. Energy harvesting mechanism is use to increase the lifetime of the network. Here it ensured that the energy balancing system works well in the sensor network.

\subsection{Network Model}

In this, model a set of sensors distributed in a geographical network. Following assumptions taken into consideration for the wireless sensor network.

- The sensor nodes are quasi stationary; this is required for sensor network applications.

- $\quad$ Links between different sensor nodes are symmetric. The transmission power level is same for all nodes.

- $\quad$ Nodes in the sensor network are self-organized.

- All sensor nodes have similar capabilities (processing/communication) and equal significance

- $\quad$ Each sensor nodes have fixed number of transmission power levels.

- Node failures are due to energy depletion of the sensor nodes.

\subsection{Clustering Criteria}

Assume that $\mathrm{n}$ nodes distributed in a field or geographical network and the above assumptions hold. Our goal is to identify a set of cluster heads, which cover the entire field or network. Each node V(i), where $1 \leq \mathrm{i} \leq \mathrm{n}$, must be mapped to exactly one cluster $C(j)$, where $1 \leq \mathrm{j} \leq \mathrm{n}(\mathrm{c})$ and $\mathrm{n}(\mathrm{c})$ is the number of clusters $(\mathrm{n}(\mathrm{c}) \leq \mathrm{n})$. A node must be able to communicate directly with its cluster head (via a single hop). Cluster heads can use a routing protocol to compute inter cluster paths for multi hop communication. The following requirements must meet:

1. Clustering is completely distributed. Each node independently makes its decisions based only on local information.

2. Clustering terminates within a fixed number of iterations (regardless of network diameter).

3. At the end of each TCP, each node is either a cluster head, or not a cluster head (which we refer to as a sensor node) that belongs to exactly one cluster. 
4. Clustering should be efficient in terms of processing complexity and message exchange.

5. Cluster heads well distributed over the sensor field or sensor network and have relatively high average residual energy compared to sensor nodes.

\subsection{CLUSTERING PARAMETERS}

The overarching goal of our approach is to prolong network lifetime. Thus cluster head selection primarily based on the residual energy of each node. Measuring this residual energy is not necessary since the energy consumed per bit for sensing, processing, and communication typically known and, hence, residual energy can estimate. To increase energy efficiency and further prolong network lifetime, we also consider intra cluster "communication cost" as a secondary clustering parameter. For example, cost can be a function of neighbor proximity or cluster density. We use the primary clustering parameter to probabilistically select an initial set of cluster heads, and the secondary parameter to "break ties" among them. A tie in this context means that a node falls within the "range" of more than one cluster head. To understand what "range" denotes in this case, observe that a node typically has a number (e.g., six) of discrete transmission power levels. Thus, the cluster range or radius is determined by the transmission power level used for intra cluster announcements and during clustering. We refer to this level as the cluster power level. The cluster power level should be set to one of the lower power levels of a node, $[15,16,17,18]$ to increase spatial reuse, and reserve higher power levels for inter cluster communication. These higher power levels should cover at least two or more cluster diameters to guarantee that the resulting inter cluster overlay will be connected. If this condition cannot satisfy, then our approach for clustering in conjunction with power level selection is inapplicable.

We analyze inter cluster connectivity conditions. The cluster power level dictates the number of clusters in our network. It is nontrivial to determine an optimal cluster power level because network topology changes due to node failures and energy depletion. The secondary clustering parameter, intra cluster communication cost, is a function of 1) cluster properties, such as cluster size, and 2 ) whether or not variable power levels are permissible for intra cluster communication. If the power level used for intra cluster communication fixed for all nodes, then the cost can be proportional to 1) node degree, if the requirement is to distribute load among cluster heads, or 2) 1 /node degree, if the requirement is to create dense clusters. This means that a node joins the cluster head with minimum degree to distribute cluster head load (possibly at the expense of increased interference and reduced spatial reuse), or joins the one with maximum degree to create dense clusters. We use the terms minimum degree cost and maximum degree cost to denote these cost types. Observe that inter cluster communication is not incorporated in the cost function since local information is insufficient in this case.

We define the average minimum reach ability power (AMRP) as the mean of the minimum power levels required by all $\mathrm{M}$ nodes within the cluster range to reach $\mathrm{u}$, if each node allowed selecting the appropriate power level to reach its cluster head, then AMRP provides a good estimate of the communication cost $[19,20,21,22]$.The AMRP of a node provides, expected intra cluster communication energy consumption, if this node becomes a cluster head. Using AMRP as cost in selecting cluster heads is superior to just selecting the closest cluster head, since it provides a unified mechanism for all nodes, including cluster heads, to break ties among tentative cluster heads. If a node has to select its cluster head among nodes not including it, the closest neighbor within its cluster range (the neighbor reached using the smallest power level) can selected as its cluster head. Table 1 summarizes the different options for computing the communication cost.

Table-1

\begin{tabular}{|l|l|l|}
\hline Goal/Power & Same & Minimum \\
\hline Load distribution & Node degree & $\begin{array}{l}\text { AMRP/ } \\
\text { Node degree }\end{array}$ \\
\hline Dense clusters & $1 /$ node degree & $\begin{array}{l}\text { AMRP/ } \\
\text { Closest node }\end{array}$ \\
\hline
\end{tabular}

\subsection{Reduction in Power Budget}

Power reduction strategies for the sensing elements of our wireless nodes summarized below:

- Turn on power to the sensor only when sampling

- Turn on power to the signal conditioning only when sampling a sensor

- Sample sensor(s) only on event

- $\quad$ Reduce the sensor sample rate to the minimum required by the application

- $\quad$ Sleep between samples

- Utilize lowest standby current electronics

- Maximize bandwidth of electronics to minimize electronics settling time

- Use fast ADC to reduce electronics and sensor "on" time

- In low data rate $(5-10 \mathrm{~Hz})$ applications, use higher power components that settle quicklyrather than

Micro power components, those settle slowly.

Power reduction strategies for the RF transceiver elements of our wireless nodes summarized below:

- $\quad$ Reduce the amount of wireless data transmitted through data compression/reduction

- $\quad$ Lower the transceiver duty cycle and frequency of data transmissions

- $\quad$ Implement strict power management - use power down and sleep modes

- Implement an event-driven transmission strategy- transmit only on sensor event(s)

\section{PROPOSED WORK}

The proposed model works on with the combined benefits of smart distributed clustering technique and energy harvesting. Routing design closely related to the network systems architecture mode and the design of routing 
protocols in WSN influenced by many challenging factors such as the sensor nodes are either equipped with global positioning system (GPS) or sensing the sink to learn about their locations, another challenge that has managed is the location of the sensors. End-to-End Delay is time taken for a one node to send packet to a sink or vice-versa. It can be either one way or round trip (packet sent from source to sink and from sink to source). Sensor nodes are deployed based on application and affect the performance of routing protocol. The nodes if randomly deployed organized themselves to establish path to acquire energy efficient and reliable connectivity network operation. In case of failures (like environmental interference, physical damage, power etc), routing protocols generate new routes to the data collection point or the destination. The number of sensor nodes deployed in the sensing area may be in the order of hundreds or thousands depending upon the routing algorithms, as they should be scalable enough to respond to the events.

\section{Algorithm for deployment of sensor nodes}

Input: Location, number of sensor nodes.

Output: $\mathrm{G}$ is a connected network including sensor nodes and base station at center.

Step-1: Deploy the sensor nodes randomly with same communication range.

Step-2: find the distance between each sensor node.

Step-3: then check the connectivity of the wireless sensor network by checking the links between each sensor node.

Step-4: Set the BS at the center of the network by calculating the midpoint of the sensor network.

Step-5: Define specific structure and color to differentiate the BS from the sensor nodes in the sensor network.

Algorithm for Dynamic cluster head selection with generalized leach

Inputs: No of sensor nodes

Area to simulate

Electronics and Amplifying charge

Energy of nodes

The position of base station

Maximum protocol simulate time

Threshold value initialized

Desired percentage of cluster heads

The number of bits transmits every time by a node, unit is Kbits

Channel bandwidth

Number of time division multiple accesses

Transit value of the node

Output: connected network including sensor nodes, cluster heads.

Step-1: Distribute all the sensor nodes in the network, energy level sent to the base station. Then divide the network into four clusters.
Step-2: Node with highest average residual energy level select as cluster head for the network.

Step-3: The communication cost calculated for each node.

Step-4: Cluster head status broadcasted and the number of cluster heads found out.

Step-5: Received signal strength indication (RSSI) is checked

Step-6: Join request send by the node to cluster head and acknowledgement message send by the cluster head to sensor node to join as the member of the cluster.

Step-7: The energy spent for choosing new cluster head is calculated.

$\underline{\text { Algorithm for distributed leach }}$

Inputs: No of sensor nodes

Area to simulate

Electronics and Amplifying charge

Initial energy of nodes

The position of base station

Maximum protocol simulate time

Threshold value initialized

Desired percentage of cluster heads

The number of bits transmits every time by a node, unit is Kbits

Channel bandwidth

Number of time division multiple accesses

Transit value of the node

Grid diameter of node

Output: connected network including sensor nodes, cluster heads.

Step-1: Distribute all the sensor nodes in the network, energy level sent to the base station. Then divide the network into four clusters.

Step-2: Node with highest residual energy level selected as cluster head for the network and node with second highest residual energy level selected as second cluster head of the particular cluster.

Step-3: The criterion for multi hops communication between cluster heads enhanced by using the grid value method.

Step-4: Cluster head status broadcasted and the number of cluster heads found out.

Step-5: Received signal strength indication (RSSI) is checked

Step-6: Join request send by the node to both cluster heads and acknowledgement message send by the cluster heads to sensor node to join as the member of the cluster.

Step-7: RSSI value calculation

Step-8: Before a sensor node becomes faulty or dead node, the node sent all the useful data to the second cluster head. Therefore, information loss controlled largely. 
Step-9: The energy spent for choosing new cluster head is calculated.

Step-10: The amount of data transmitted between the nodes calculated according to the number of nodes present in the network.

Algorithm for energy harvesting leach

Inputs: No of sensor nodes

Area to simulate

Electronics and Amplifying charge

Initial energy of nodes

The position of base station

Maximum protocol simulate time

Threshold value initialized

Desired percentage of cluster heads

The number of bits transmits every time by a node, unit is Kbits

Channel bandwidth

Number of time division multiple accesses

Transit value of the node

Grid diameter of node

Harvested Energy Amount for each node

Response time for harvesting energy

Output: connected network including sensor nodes, cluster heads.

Step-1: Distribute all the sensor nodes in the network, energy level sent to the base station. Then divide the network into four clusters.

Step-2: Node with highest residual energy level selected as cluster head for the network and node with second highest residual energy level selected as second cluster head of the particular cluster.

Step-3: The criterion for multi hops communication between cluster heads enhanced by using the grid value method.

Step-4: Cluster head status broadcasted and the number of cluster heads found out.

Step-5: Received signal strength indication (RSSI) is checked

Step-6: Join request send by the node to both cluster heads and acknowledgement message send by the cluster heads to sensor node to join as the member of the cluster.

Step-7: RSSI value calculation

Step-8: Before a sensor node becomes faulty or dead node, the node sent all the useful data to the second cluster head. Therefore, information loss controlled largely.

Step-9: The harvested energy for each node is calculated.

Step-10: The response time for energy harvesting calculated by considering the distance of sensor node and harvesting system present in the network.
Step-11: energy harvesting can be possible through energy storage system.

Step-12: The energy spent for choosing new cluster head is calculated.

Step-13: The communication cost calculated for the network.

\section{SIMULATION AND RESULTS}

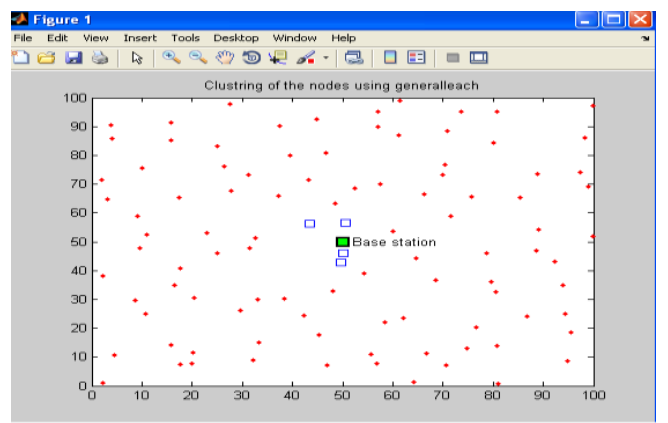

Fig 4.1General leach implementation

\begin{tabular}{|c|c|}
\hline Simulation Parameters & Values \\
\hline Network area & $100 \mathrm{~m} * 100 \mathrm{~m}$ \\
\hline Number of nodes & 100 \\
\hline Initial Energy & $0.5 \mathrm{~J}$ \\
\hline Percentage of cluster head & 0.5 \\
\hline Base Station Position & $50 \mathrm{~m} * 50 \mathrm{~m}$ \\
\hline Eelec & $50 \mathrm{~nJ} / \mathrm{bit}$ \\
\hline Etx=Erx & $50 \mathrm{~nJ} / \mathrm{bit}$ \\
\hline Eamp & $10 \mathrm{pJ} / \mathrm{bit}$ \\
\hline Packet size & $2000 \mathrm{bit}$ \\
\hline Rounds & 1000 \\
\hline
\end{tabular}

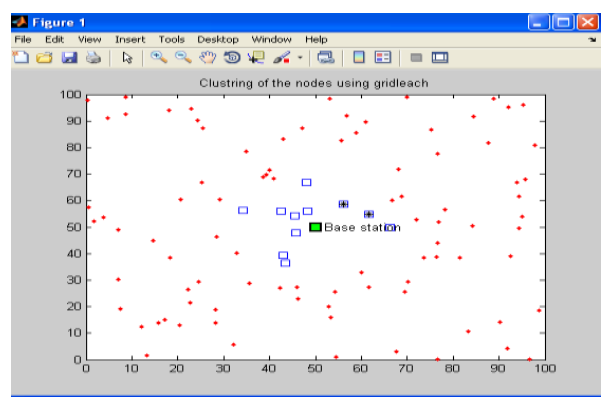

Fig 4.2 Grid leach implementation

\begin{tabular}{|c|c|}
\hline Simulation Parameters & Values \\
\hline Network area & $100 \mathrm{~m} * 100 \mathrm{~m}$ \\
\hline Number of nodes & 100 \\
\hline Initial energy & $0.02 \mathrm{~J}$ \\
\hline Percentage of cluster head & 0.1 \\
\hline Base Station Position & $50 \mathrm{~m} * 50 \mathrm{~m}$ \\
\hline
\end{tabular}




\begin{tabular}{|c|c|}
\hline Efs & $10 \mathrm{~nJ} / \mathrm{bit}$ \\
\hline Etx=Erx & $50 \mathrm{~nJ} / \mathrm{bit}$ \\
\hline Eda & $5.0 \mathrm{~nJ} / \mathrm{bit}$ \\
\hline Packet size & $2000 \mathrm{bit}$ \\
\hline Rounds & 1000 \\
\hline $\mathrm{cm}$ & 30 \\
\hline dm & 3000 \\
\hline grid value & $10 \mathrm{~m}$ \\
\hline
\end{tabular}

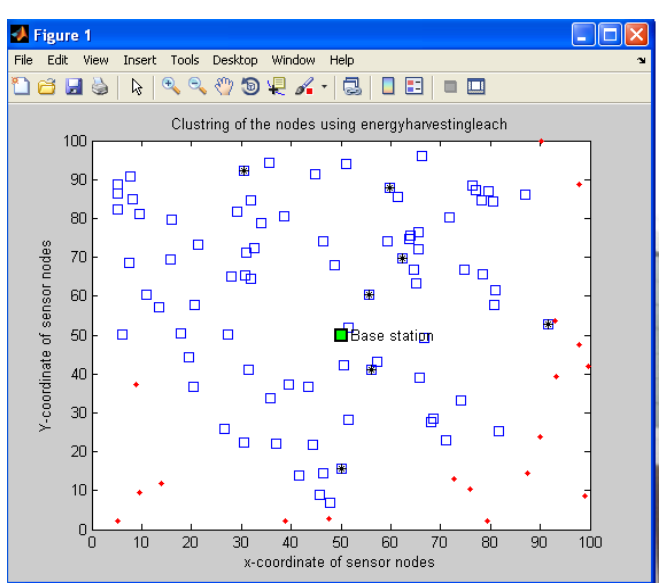

Fig 4.3 Energy harvesting leach implementation

\begin{tabular}{|c|c|}
\hline Simulation Parameters & Values \\
\hline Network area & $100 \mathrm{~m} * 100 \mathrm{~m}$ \\
\hline Number of nodes & 100 \\
\hline Initial energy & $0.02 \mathrm{~J}$ \\
\hline Percentage of cluster head & 0.1 \\
\hline Base Station Position & $50 \mathrm{~m} * 50 \mathrm{~m}$ \\
\hline Efs & $10 \mathrm{~nJ} / \mathrm{bit}$ \\
\hline Etx=Erx & $50 \mathrm{~nJ} / \mathrm{bit}$ \\
\hline Eda & $5.0 \mathrm{~nJ} / \mathrm{bit}$ \\
\hline Packet size & $2000 \mathrm{bit}$ \\
\hline Rounds & 1000 \\
\hline cm & 30 \\
\hline dm & 3000 \\
\hline grid value & $10 \mathrm{~m}$ \\
\hline Harvesting energy & $20 \mathrm{~nJ} / \mathrm{node}$ \\
\hline Response time & $0.1 \mathrm{~ms}$ \\
\hline
\end{tabular}

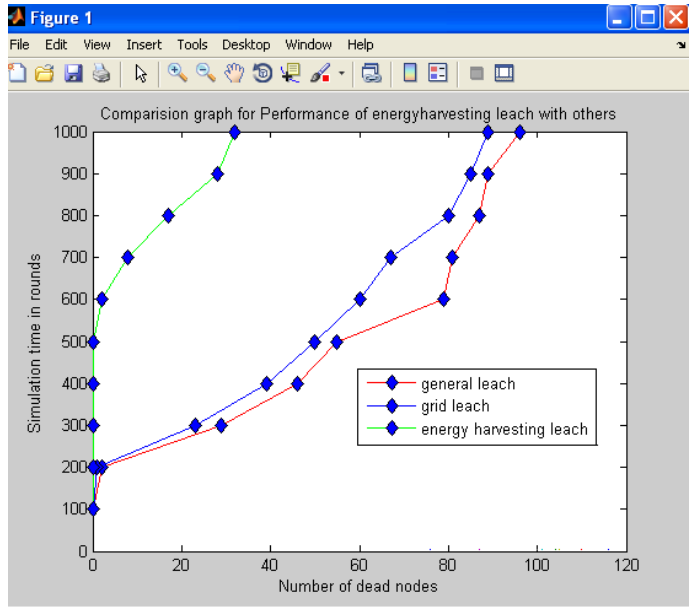

Fig 4.4 Comparison graph for performance level of different protocols

From the comparison graph, we can say that energy harvesting leach can outperforms as compared to general leach and grid leach. So the proposed model enhanced the lifetime of the sensor network to a greater level, which is the real motto in current WSN scenario.

\section{CONCLUSION}

As we know, in wireless sensor networks; improving lifetime of the network is a very challenging issue. So here the presented energy harvesting leach model significantly increases the lifetime of the network that level seems to be beyond the excepted level. At the same time the effective clustering technique balances the energy budget and also maintains the fault tolerance capability of the network. So the presented model is really better than the existing models in all respect.

\section{REFERENCES}

[1] Lee Myeong-Hyeon, Choi Yoon-Hwa. Fault detection of wireless sensor networks. Computer Communications 2008;31:3469-75

[2] Bari Ataul, et al. Design of fault tolerant wireless sensor networks satisfying survivability and lifetime requirements. Computer Communications 2012;35: 320-33.

[3] Challal Y, et al. Secure and efficient disjoint multipath construction for fault tolerant routing in wireless sensor networks. Journal of Network and Compu- ter Applications 2011;34:1380-97.

[4] Chen Xian, et al. Fault-tolerant monitor placement for out-of-band wireless sensor network monitoring. Ad Hoc Networks 2012;10:62-74.

[5] D.D. Geeta , N.Nalini , RajashekharC.Biradar. Fault tolerance in wireless sensor network using hand-off and dynamic power adjustment approach, Journal of Network and Computer Applications 36 (2013) 11741185

[6] B. Shirazi, A.R. Hurson, and K.M. Kavi, Scheduling and Load Balancing in Parallel and Distributed Systems. IEEE CS Press, 1995.

[7] C. Intanagonwiwat, R. Govindan, and D. Estrin, "Directed Diffusion: A Scalable and Robust Communication Paradigm for Sensor Networks," 
Proc. ACM/IEEE Int'l Conf. Mobile Computing and Networking (MOBICOM), 2000.

[8] J. Kulik, W. Heinzelman, and H. Balakrishnan, "Negotiation- Based Protocols for Disseminating Information in Wireless Sensor Networks\}," ACM Wireless Networks, vol. 8, nos. 2-3, pp. 169-185, 2002. citeseer.nj.nec.com/kulik99negotiationbased. Html.

[9] J.-H. Chang and L. Tassiulas, "Energy Conserving Routing in Wireless Ad-Hoc Networks," Proc. IEEE INFOCOM, Mar. 2000.

[10] W. Heinzelman, A. Chandrakasan, and H. Balakrishnan, "An Application-Specific Protocol Architecture for Wireless Microsensor Networks," IEEE Trans. Wireless Comm., vol. 1, no. 4, pp. 660670, Oct. 2002.

[11] K. Akkaya, M. Younis, A survey of routing protocols in wireless sensor networks, Elsevier Ad Hoc Network Journal 33 (2005) 325-349.

[12] V. Mhatre, C. Rosenberg, Energy and cost optimizations in wireless sensor networks: a survey, in: A. Girard, B. Sanso, F. Vazquez-Abad (Eds.), Performance Evaluation and Planning Methods for the Next Generation Internet, Kluwer Academic Publishers, 2005, pp. 1-23, chap. 1

[13] C.P. Townsend, S.W. Arms, Wireless Sensor Networks: Principles and Applications, Sensor Technology Handbook, Editor:Jon S. Wilson, publisher: Elsevier Newnes, ISBN: 0-7506-7729-5, Chapter 22, pp. 575-589, 2005

[14] Ossama Younis, Student Member, IEEE, and Sonia Fahmy, Member, IEEE HEED: A Hybrid, EnergyEfficient,Distributed Clustering Approach for Ad Hoc Sensor Networks.IEEE transactions on mobile computing, vol. 3, no. 4, october-december 2004.
[15] Xingfa Shen , Cheng Bo , Jianhui Zhang , Shaojie Tang, Xufei Maob, Guojun Dai.EFCon: Energy flow control for sustainable wireless sensor networks.In press.

[16] Satvir Singh, Meenaxi, A Survey on Energy Efficient Routing in Wireless Sensor Networks, Volume 3, Issue 7, July 2013.

[17] Z. A. Eu, H.-P. Tan, W. K. G. Seah., Routing and Relay Node Placement in Wireless Sensor Networks Powered by Ambient Energy Harvesting IEEE 2009

[18] S. Yi, J. Heo, Y. Cho, J. Hong., PEACH: Powerefficient and adaptive clustering hierarchy protocol for wireless sensor networks ELSEVIER Computer Communications 30 (2007) 2842-2852

[19] A. Manjeshwar and D. P. Agrawal., TEEN: A Routing Protocol for Enhanced Efficiency in Wireless Sensor Networks IEEE 2001

[20] J. Zhao, A. T. Erdogan., A Novel Self-organizing Hybrid Network Protocol for Wireless Sensor Networks Proceedings of the First NASA/ESA Conference on Adaptive Hardware and Systems (AHS'06) 0-7695-2614-4/06 2006 IEEE.

[21] R. C. Shah and J. M. Rabaey., Energy Aware Routing for Low Energy Ad Hoc Sensor Networks IEEE wireless Communications and Networking Conf. (WCNC), March 17-21, 2002, Orlando, FL.

[22] R. Gómez Cid-Fuentes, E. Alarcón and A. Cabellos-Aparicio, Energy harvesting enabled Wireless sensor networks, NaNoNetworking Summit 2012.

[23] Z.J. Haas, M.R. Pearlman, and P. Samar, "The Zone Routing Protocol (ZRP) for Ad-Hoc Networks," Internet Draft, draft-ietfmanet-zone-zrp-04.txt, July 2002. 